\title{
3D Brownian Motion Thin Film Fluid spraying nanoparticles impact of Convective Heat Phenomena over Stretchable Rotating Surface: Numerical Computation
}

\author{
Zeeshan Khan ( $\nabla$ zeeshansuit@gmail.com ) \\ Bacha Khan University Charsadda \\ Haroon Rasheed \\ Abdul Wali Khan University \\ Waris Khan \\ Hazara University Mansehra
}

\section{Research Article}

Keywords: RK4 and HAM, Nanomaterial flow, Brownian motion, 3D model, Convection conditions, Stretchable rotating disc

Posted Date: August 31st, 2021

DOl: https://doi.org/10.21203/rs.3.rs-850329/v1

License: (c) (1) This work is licensed under a Creative Commons Attribution 4.0 International License.

Read Full License 
3D Brownian Motion Thin Film Fluid spraying nanoparticles impact of Convective Heat Phenomena over Stretchable Rotating Surface: Numerical Computation

\author{
Zeeshan Khan*1, Haroon Ur Rasheed ${ }^{2}$,Waris Khan ${ }^{3}$ \\ ${ }^{1}$ Department of Mathematics and Statistics, Bacha Khan University Charsadda, 24420, KP, Pakistan \\ ${ }^{2}$ Department of Mathematics, Abdul Wali Khan University, Mardan 23200, KP, Pakistan \\ ${ }^{3}$ Department of Mathematics and Statistics, Hazara University Mansehra, 21120 KP, Pakistan \\ *Corresponding author email: zeeshansuit@gmail.com
}

\begin{abstract}
The purpose of this research is to examine thin-film nanomaterial movement in three dimensions over a stretchable rotating inclined surface. Similarity variables are used to transform fundamental systems of equations into a set of First-order Differential Equations. The Runge-Kutta Fourth Order approach is utilized for numerical purpose solution. Variable thickness., Unsteadiness parameter., Prandtl number., Schmidt number., Brownian-motion parameter., and Thermophoretic parameter have all been seen to have an impact. Physically and statistically, the indispensable terms namely Nusselt as well as Sherwood numbers are also investigated. As the dimensionless factor $S$ grows, the temperature field decreases. The momentum boundary layer is cooled when the parameter $S$ is improved, and the opposite effect is observed for Nusselt number. A greater Schmidt number Sc reduces the Sherwood number by increasing the kinematic viscosity as well as Concentration of the chemical species. Further, the RK4 method is also validated with the HAM approach. Furthermore, we verified the acquired results by establishing a comparison with previous literature, and we discovered an outstanding match, confirming the accuracy of the current communication.
\end{abstract}

Keywords: RK4 and HAM, Nanomaterial flow, Brownian motion, 3D model, Convection conditions, Stretchable rotating disc.

\title{
1. Introduction
}

In the sphere of chemistry and applied sciences, the development of liquid condensate from a cool, saturated vapor is crucial. Many researchers have looked into this phenomenon under a variety of circumstances. Gregg et al. [1] used the centrifugal force characteristic on a cold spinning disc to investigate the removal of moisture. They converted the fundamental flow equations into highly nonlinear equations and attempted 
the numerical solution for liquid layer thicknesses of finite and varying thickness. Hudson et al. [2] carried their work a step further by incorporating vapour drag. The theory of Sarma et al. [3] has been expanded to include the adhesion term at the plate surface.

The mutual fluid, which has a poor thermal conductivity, is employed as a basis fluid in much of the available literature. The outputs of these types of heat systems are extremely low. Nanoparticles are tiny particles that are injected inside the base fluid to improve the chemical property of consideration fluid. Hatami [4] investigated the discharge of a nanomaterial across a revolving, inclined plane. Significant physical results for cooling purposes were preserved.

The application of time-dependent flow field in engineering and physical science is equally significant. In situation of porous medium, Attia. [5] examined the flow behavior around a circular cylinder. The flow rate through a porous medium was examined by Ishak [6]. They employed Nano fluids to heat transfer. The numerical findings of unsteady magnetohydrodynamic streaming of a flow of Nano fluid across a permeable upwardly expanding medium were observed by Rashidi et al. [7]. Makinde et al. [8] observed the impact of changing viscosity on nanoliquid streaming. Akbar et al. [9] used a magnetism to analyze a two dimensional stream of a nanomaterial and used shooting approach to find the numerical solution. During partial slip scenarios, Chung et al.[10] analyzed micro-polar nanomaterial moment generated by rotating disc. We can look at the most recent works [11-13] for a thorough analysis as well as in investigation of magnetohydrodynamic nano fluid streaming with various properties. The particles employed in Nano fluids are often made up of atoms (Al., Cu), oxides (Al2O3), nitrides (AlN., SiN)., or thermoplastic elements (Polymer, Graphene oxide), with a propagation liquid such as water as well as ethylene glycol serving as the conventional fluids. As a base lubricant, oily chemicals, biofluids, and polymer coatings can be employed. Nanomaterials have sizes between 1 and 100 nanometers. Nano fluids typically include upto $5 \%$ aspect ratio of nanomaterial's to confirm greater heat-transfer improvements. Nano fluids have peculiar properties that allow them to be used in a variety of applications., involving hypervisor engines., pharmaceutical procedures., fuel cells., integrated circuits., as well as heat transmission. These have been extensively utilized in 
engineering-applications for ship soil in boilers exhaust gases heat dissipation and defense, as well as in spaceflight, grinding, nuclear plant and pressurizer, chiller, residential refrigerator, and engines vehicle plasma management. Nano fluids greatly improve the convection of the polymer matrix, hence researchers are particularly interested in studying the flow with Nano fluids. Nanoparticles are also exceedingly consistent, with no additional problems like pressure decrease, erosion, or sedimentation. Choi [14] was the first to develop Nano fluids technology. Thermodynamic management is a challenge in ferromagnetic materials and ultrafast computing applications. Rheological characteristics of Nano fluids have been a focus of interest and research numerous applications in electronic freezing and heat transmission. Xie et al. [15] measured the heat capacity as well as viscous of ethylene polyvinyl Nano fluids to investigate their thermal transport capabilities. Xie et al.[16] explored the rheological impacts as well as transmission of heat characteristics of A12O3 Nano fluids focusing on 45 percent ethylene glycol and 55 percent water in another investigation. Carbon-based nanostructures have gained popularity since the development of nanotubes (CNTs) in 1991 because to their distinctive facial, radioactive, physical, and electromagnetic capabilities. Yu et al. [17] used a green technique to manufacture silver nanoparticle-decorated intra Nano fluids (MWNT) blends (Ag-NPs). A single layer of graphite, of 2D form of carbon, has been discovered to exhibit good crystal quality and efficient electrical transport at ambient temperature in the event of graphene. According to Xie and Chen [18] it has evolved into a remarkable material with unusual physical, biological, and structural features. Yu et al. [19] found that Nano fluid including grapheme oxide nanoparticles have much greater thermodynamic properties than the base fluid. Because of the various potential applications, it is critical to learn further about heat exchange characteristics of water. Among the most contentious issues in hybrid Nano fluid is efficient thermal diffusivity. Furthermore, due to the complexity and variability, physical nature is still poorly understood. Brownian motion generated convection and efficient transmission through propagating nanoparticle routes are indeed the most two common mechanisms for increased heat transmission in Nano fluids, according to a rigorous investigation. While its effects of various parameters like nanotube concentration and aggregate intensity have been studied in literatures [20-23], yet an 
entire mechanistic explanation is not provided. The exceedingly intricate processes of heat transmission and the interplay involving thermal conductivity, pore size with nanoparticle concentration make it difficult to evaluate the actual thermophysical properties analytically. As a result, the precise mechanism of convection in Nano fluids is currently unknown. To close this gap, Khan [24] used the customs and beliefs to establish analytical formulas for optimal nanoparticle concentration while accounting heat convection due to Brownian moment of nanomaterials. Analogously, Mustafa et al. [25] focused into the progress of nanoparticle survey by incorporating fractal theory as well as conveying a nonlinear model based on the fractal dispersion and heat condensation for nanomaterial fluid owing to Brownian moment of nanomaterial's. A thorough explanation of the Nano fluid was addressed by Akbar and Nadeem [26]. Nowar [27] investigated Maxwell fluid's effect and variable viscosity for non-Newtonian flow of Nano fluid in a pipe. Choi et al. [28] described how a Nano fluid passes over a stretched surface. Terekhov et al. [29] investigated wall behavior impact at peristaltic moment of a ferrofluid. Endoscopic examination of peristaltic nanomaterial moment was addressed via $\mathrm{Yu}$ et al. [30]. In domain of Hall current with permeable medium, Hojjat [31] addressed Nano fluid model for peristaltic flow. The sources [32-40] contain a comprehensive investigation on various aspects of Nano fluids.

A electromagnetic Nano fluid is a unique substance that combines the properties of a fluid as well as a magnetic material. Such fluids are used in a variety of applications, including magneto optical wavelength filters as well as other optical materials such as complicated structures and tunable fiber filters, glass panels, and switches. Changing the amplitude of a magnetic field can modify a variety of physical features of all such fluids. Nano fluids based on magnetism are currently being used in a variety of fields, including, biomedicine, pharmacy, and submarine float isolation. In most of biomedical applications which involves Nano fluids, such as drug delivery, magnetic detection, and significant decline in neuroimaging. Because of its application in power sources, MHD accelerator, refrigerated coils, transmission system, electro transformers, and heaters, MHD processes are important. Because of the richness of this concept, various scholars have focused on MHD motions. Transfer of energy is facilitated by composition gradients, while mass movement is facilitated by a thermal gradient. These characteristics 
of MHD motion are used in fluid mechanics for suspension and fluid pump, liquids actuators, and transpiration techniques, as well as aerodynamics. Heat transmission in boundary layer flow via stretched surfaces has several applications in the injection molding. The MHD motions inside an electrical conductor liquid, which may manage the impact of cooling, effectively accomplish the quality level of a manufacturing process. Several technological operations, such as glass fiber production, foodstuff and paper production, glass blowing, metal spinning, and metallurgical procedures including crystal production, polyester \& rubber sheet preparation, bronze threads enameling and decorating, and many more are significant manufacturing implementations of the problem of viscoelastic mobility and heat transmission beyond a stretched surface. During the production of such sheets, the problems in molten state are stretched from either a gap to reach the appropriate size. The finished product mostly with needed qualities is manufactured due to temperature stretching rate throughout the procedure as well as the stretching cycle. Abu [41] investigated Brownian motion and thermal flexibility impacts on MHD viscoelastic moment of a nanomaterial via a stretchable porous material. Alim et al. [42] examine the MHD time dependent motion of a Nano liquid through a longitudinal stretch sheet under suction/injection. There's been enough investigation on displacement past stretched surfaces. Khan [43] was the first one to address flow on smooth and substantial continuous surfaces. Gul et al. [44] looked at the fluid flow an extendable barrier by assuming that only the surface velocity varied linearly from the slit. MHD solution of a viscoelastic-non-Newtonian liquid over a stretching disc was discovered by Gul et al.[45]. Aziz et al. [46] studied the impact of varied Al2O3 water Nano liquid characteristics on the enhancement of heat transmission in entropy generation. Khan et al. [47] studied heat generation using a flat - plate absorber and a nanofluids with changeable characteristics. In study, thin fluids film fluxes are receiving a lot of attention. Variable parameters of a thin fluid flow through a extending region was studied by Qasim et al. [48]. Prashant et al. [49] intensively reviewed the flow as well as heat transmission non-Newtonian liquid via a porous media through shrinking surface. Gireesha et al. [50] explored thermophoresis and nonlinear thermal MHD thin film fluid of second grade with temperature dependent viscosity through a stretched sheet, as well as heat and mass transport. Time-dependent stretched surface with heat generation, Wang 
[51] observed thin film flow and heat source. Nazar et al. [52] investigated Williamson nanomaterial fluid with changing concentration and temperature on the time-dependent stretched. Adopting model of Buongiorno, Emam et al. [53] investigated the transmission of pressure drop and heat in nanofluids through a time-dependent stretch sheet. Emam et al. [54] demonstrated heat transfer over magnetic field using time-dependent stretch sheet along thermal radiation of thin flow. The flow phenomena and heat transmission past small cylinders has undergone a significant revolution in recent years. The unavoidable need for slim devices that reduce drag while delivering whole lift in order to keep the body afloat in specific scenarios. In narrow cylinder., radius can be like the boundary layer thickness having is axisymmetric behavior rather than two-dimensional, and the governing equation includes a transverse curve that impacts the temperature and velocity fields through force.

A normal curvature has an effect on coefficient of skin friction and rate of heat transfer at the wall, which is relevant to this concept. The preparation of combustion chambers, chimney stacks, coolers, offshore structures, thin film deposition, and paper manufacture are all examples of flow past a cylinder and related transfer of heat properties. Sheikoleslami et al. [55] was the one to evaluate the third-grade non-linear viscous fluid flow via a stretched circular tube. Ahmad et al. [56] concentrate the fluid motion beside a extending tube utilizing Keller-box approach for solution. The analogous results of the natural convection investigation over a quasi-stretched cylinder were obtained by Sheikholeslami [57]. Wang [58] provided a computational solution of MHD Newtonian fluid moment through a stretched disc. Nanomaterial mobility with heat as well as attractive field over extending surface was described by Kleinstreuer et al. [59]. Koo [60] looked at how incompressible Newtonian fluid moves and heat transfer across a stretchable cylinder with variable viscosity. Prasher et al. [61] examined effect of uniform pressure over stretchable cylinder in presence of nanoparticles liquid. Jang [62] focused at simulated fluid flow including heat transfer within micro systems. Other studies of nanofluids and many intriguing challenges with regard to various features can be found in [63-66].

The goal of this work is to investigate the spraying nanomaterial fluid across an angled rotating disc for cooling purposes, in light of the preceding critical debate. Through 
suitable transformations, the basic equations of Continuity., Momentum., Thermal Boundary layer., as well as mass for time dependent density flow are rehabilitated to Non-linear Ordinary Differential Equations. To generate first ODEs, these are additionally distorted in order to obtain numerical solution. The numerical solution of the transformed First ODE was achieved using the RK4 technique. The numerical results are indeed validated using the HAM for the sake of confirmation. Furthermore, we verified the acquired results by establishing a comparing with previous literatures, and we discovered an outstanding match, confirming the accuracy of the current communication
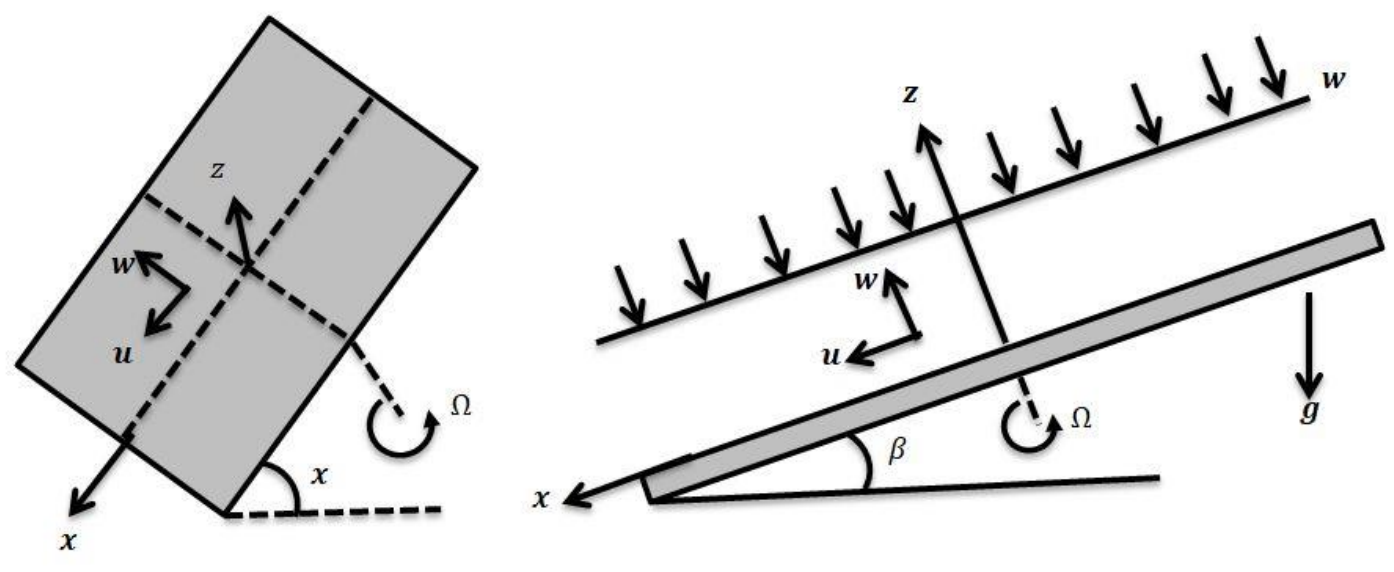

Figure. 1. Geometry of the problem.

\section{Modeling of the Problem}

Take a rotating disc with a $3 \mathrm{D}$ unsteady nanomaterial thin-film moment. As seen in figure 1, the disc rotates with angle $\Omega$. The horizontal line has been at an inclination $\beta$ with the slanted disc. The nanomaterial sheet thickness is denoted by $h$, as well as the spray speed is indicated by $W$. Because the fluid film's thickness is already so thin in comparison to the radius of the disc, the terminal effect is neglected. The gravitation force $\bar{g}$ is exerting in the negative direction as it often does. The temperature $\theta_{0}$ is at the film surface, whereas $\theta_{w}$ is over the disc. The Concentration happening on surface film is $C_{0}$, while the concentration on surface of is $C_{h}$. 
The constant pressure $p_{0}$ held at surface film, is merely the function of z. Omitting viscous dissipation, and for unsteady flow essential model equations for continuity., momentum boundary layer., temperature boundary layer as well as mass are provided

$$
\begin{aligned}
& \frac{\partial u}{\partial x}+\frac{\partial v}{\partial y}+\frac{\partial w}{\partial z}=0, \\
& \rho_{f}\left(\frac{\partial u}{\partial t}+u \frac{\partial u}{\partial x}+v \frac{\partial u}{\partial y}+w \frac{\partial u}{\partial z}\right)=\mu\left(\frac{\partial^{2} u}{\partial x^{2}}+\frac{\partial^{2} u}{\partial y^{2}}+\frac{\partial^{2} u}{\partial z^{2}}\right)+\rho_{f} \bar{g} \sin \beta \\
& \rho_{f}\left(\frac{\partial v}{\partial t}+u \frac{\partial v}{\partial x}+v \frac{\partial v}{\partial y}+w \frac{\partial v}{\partial z}\right)=\mu\left(\frac{\partial^{2} v}{\partial x^{2}}+\frac{\partial^{2} v}{\partial y^{2}}+\frac{\partial^{2} v}{\partial z^{2}}\right) \\
& \rho_{f}\left(\frac{\partial w}{\partial t}+u \frac{\partial w}{\partial x}+v \frac{\partial w}{\partial y}+w \frac{\partial w}{\partial z}\right)=\mu\left(\frac{\partial^{2} w}{\partial x^{2}}+\frac{\partial^{2} w}{\partial y^{2}}+\frac{\partial^{2} w}{\partial z^{2}}\right)-\rho_{f} \bar{g} \cos \beta-p_{z}, \\
& \frac{\partial \theta}{\partial t}+u\left(\frac{\partial \theta}{\partial x}\right)+v\left(\frac{\partial \theta}{\partial y}\right)+w\left(\frac{\partial \theta}{\partial z}\right)=\alpha\left(\frac{\partial^{2} \theta}{\partial x^{2}}+\frac{\partial^{2} \theta}{\partial y^{2}}+\frac{\partial^{2} \theta}{\partial z^{2}}\right) \\
& \quad\left(\rho c_{p}\right)_{p}\left[D_{B}\left\{\frac{\partial C}{\partial x} \cdot \frac{\partial \theta}{\partial x}+\frac{\partial C}{\partial y} \cdot \frac{\partial \theta}{\partial y}+\frac{\partial C}{\partial z} \cdot \frac{\partial \theta}{\partial z}\right\}+\frac{D_{\theta}}{\theta}\left\{\left(\frac{\partial \theta}{\partial x}\right)^{2}+\left(\frac{\partial \theta}{\partial y}\right)^{2}+\left(\frac{\partial \theta}{\partial z}\right)^{2}\right\}\right] \\
& \frac{\partial C}{\partial t}+u\left(\frac{\partial C}{\partial x}\right)+v\left(\frac{\partial C}{\partial y}\right)+w\left(\frac{\partial C}{\partial z}\right)=D_{B}\left(\frac{\partial^{2} C}{\partial x^{2}}+\frac{\partial^{2} C}{\partial y^{2}}+\frac{\partial^{2} C}{\partial z^{2}}\right)+\left(\frac{D_{\theta}}{\theta_{0}}\right)\left(\frac{\partial^{2} \theta}{\partial x^{2}}+\frac{\partial^{2} \theta}{\partial y^{2}}+\frac{\partial^{2} \theta}{\partial z^{2}}\right),
\end{aligned}
$$

With boundary conditions

$$
\begin{array}{r}
u=-\Omega y, v=-\Omega x, w=0, \theta=\theta_{w}, C=C_{h}, \\
u_{z}=0, v_{z}=0, w=-W, \theta=\theta_{0}, C=C_{0}, p=p_{0},
\end{array}
$$

We assume the transformations

$$
\begin{aligned}
& u=\frac{-\Omega y}{1-b t} g(\eta)+\frac{\Omega x}{1-b t} f^{\prime}(\eta)+\frac{\bar{g}}{\sqrt{1-b t}} k(\eta) \sin \frac{\beta}{\Omega^{\prime}}, \\
& v=\frac{-\Omega x}{1-b t} g(\eta)+\frac{\Omega y}{1-b t} f^{\prime}(\eta)+\frac{\bar{g}}{\sqrt{1-b t}} h(\eta) \sin \frac{\beta}{\Omega^{\prime}}, \\
& w=-2 \sqrt{\frac{\Omega v_{f}}{1-b t}} f(\eta), \eta \theta(\eta)=\frac{\theta-\theta_{w}}{\theta_{0}-\theta_{w}}, \eta \phi(\eta)=\frac{C-C_{w}}{C_{0}-C_{w}} . \\
& \eta=z \sqrt{\frac{\Omega}{v_{f}(1-b t)}}
\end{aligned}
$$

The transformations described in Eq. (8) are then placed into Eqs. (2)-(7), resulting in Eq. (1) being confirmed similarly and Eq. (2)-(6) yielding:

$$
\begin{aligned}
& f^{\prime \prime \prime}-\left(f^{\prime}\right)^{2}+g^{2}-2 f f^{\prime \prime}-S\left(f^{\prime}+\frac{\eta}{2} f^{\prime \prime}\right)=0, \\
& K^{\prime \prime}-K f^{\prime}-h g+2 f K^{\prime}+1-\frac{s}{2}\left(K+\eta K^{\prime}\right)=0, \\
& g^{\prime \prime}-2 g f^{\prime}+2 g^{\prime} f-S\left(g+\frac{\eta}{2} g^{\prime}\right)=0, \\
& h^{\prime \prime}-K g-h f^{\prime}+2 f h^{\prime}-\frac{s}{2}\left(h-\eta h^{\prime}\right)=0 .
\end{aligned}
$$


Equations (5) and (6) become if $\theta$ and $C$ are functions of $\mathrm{z}$

$$
\begin{aligned}
& \theta^{\prime \prime}+2 \operatorname{Pr} f \theta^{\prime}+N b \phi^{\prime} \theta^{\prime}+N t\left(\theta^{\prime}\right)^{2}+\frac{s}{2}\left(\eta \theta^{\prime}+\eta^{2} \theta^{\prime \prime}\right)=0, \\
& \phi^{\prime \prime}+2 S c f \phi^{\prime}+\frac{N t}{N b} \theta^{\prime \prime}+\frac{s}{2}\left(\eta \phi^{\prime}+\eta^{2} \phi^{\prime \prime}\right)=0, \\
& f(0)=0, f^{\prime}(0)=0, f^{\prime \prime}(\delta)=0, g(0)=0, g^{\prime}(\delta)=0, \\
& K(0)=0, K^{\prime}(\delta)=0, h(0)=0, h^{\prime}(\delta)=0 \\
& \theta(0)=0, \theta(\delta)=1, \phi(0)=0, \phi(\delta)=1 .
\end{aligned}
$$

Where

$\operatorname{Pr}=\frac{\mu}{\rho_{f}}, S c=\frac{\mu}{\rho_{f} D}, N b=\frac{(\rho c)_{p} D_{b}\left(C_{h}\right)}{\left\{(\rho c)_{f}\right\}}, N t=\frac{(\rho c)_{p} D_{\theta}\left(\theta_{H}\right)}{\left\{(\rho c)_{f} \theta_{c}\right\}}, S=\frac{1}{\Omega}$.

$\delta$ is thickness of normalized.

$\delta=\varepsilon \sqrt{\frac{\Omega}{v_{f}(1-b t)}}$.

Eq. (17) can be calculated using spraying velocity

$$
f(\delta)=\frac{w}{2 \sqrt{\Omega v}}=\alpha
$$

Integrating equation (4), we get the pressure

For exact solution take $\operatorname{Pr}=0$ by utilizing $\theta(\delta)=1$, we get

$\theta^{\prime}(0)=\frac{1}{\delta}$

The dimensionless Nusselt and Sherwood numbers

$$
\begin{aligned}
& N u=\frac{\left(\frac{\partial \theta}{\partial z}\right)_{w}}{\theta_{0}-\theta_{w}}=\delta \theta^{\prime}(0) . \\
& S h=\frac{\left(\frac{\partial C}{\partial z}\right)_{w}}{C_{0}-C_{w}}=\delta \phi^{\prime}(0) .
\end{aligned}
$$

\section{Numerical and HAM solutions}

Equation (22) is incorporated to [9 - 14] in order to obtain first ODE

$$
\begin{aligned}
& y_{1}=\eta, y_{2}=f, y_{3}=f^{\prime}, y_{4}=f^{\prime \prime}, y_{5}=g, y_{6}=g^{\prime}, y_{7}=K, y_{8}=K^{\prime}, \\
& y_{9}=h, y_{10}=h^{\prime}, y_{11}=\theta, y_{12}=\theta^{\prime}, y_{13}=\phi, y_{14}=\phi^{\prime} .
\end{aligned}
$$

Utilizing equation (22), the nonlinear higher order derivative system (9-14) are transformed to the first-order ODEs system [22-28] which are given below

$$
\begin{aligned}
& \mathrm{y}_{4}^{\prime}=\left(y_{3}\right)^{2}-y_{5}^{2}-2 y_{2} y_{4}+S\left(y_{3}+\frac{1}{2} y_{1} y_{4}\right), \\
& \mathrm{y}_{6}^{\prime}=2 y_{5} y_{3}-2 y_{6} y_{2}+S\left(y_{5}+\frac{1}{2} y_{1} y_{6}\right),
\end{aligned}
$$




$$
\begin{aligned}
& \mathrm{y}_{8}^{\prime}=y_{7} y_{3}-y_{9} y_{5}-2 y_{2} y_{8}-1+\frac{s}{2}\left(y_{7}+y_{1} y_{8}\right), \\
& \mathrm{y}_{10}^{\prime}=y_{7} y_{5}+y_{9} y_{3}-2 y_{2} y_{10}-\frac{s}{2}\left(y_{9}-y_{1} y_{10}\right), \\
& \mathrm{y}_{12}^{\prime}=-2 \operatorname{Pr} y_{2} y_{12}-N b y_{14} y_{12}-N t y_{12} y_{12}-\frac{s}{2}\left(\left(y_{1}\right)^{2} y_{12}+y_{1} y_{11}\right), \\
& \mathrm{y}_{14}^{\prime}=-2 S c y_{2} y_{14}-\frac{N t}{N t}\left(-2 \operatorname{Pr} y_{2} y_{12}-N b y_{14} y_{12}-N t y_{12}^{2}\right)-\frac{s}{2}\left(\left(y_{1}\right)^{2} y_{14}+y_{1} y_{13}\right) .
\end{aligned}
$$

The initial conditions for the nanomaterial fluid flow by applying equation (22) are

$$
\begin{array}{cl}
y_{1}=0, & y_{2}=0, y_{3}=0, y_{4}=u_{1}, y_{5}=1, y_{6}=u_{2}, y_{7}=0, y_{8}=u_{3}, y_{9}=0, \\
& y_{10}=u_{4}, y_{11}=0, y_{12}=u_{5}, y_{13}=0, y_{14}=u_{6} .
\end{array}
$$

For solution of differential equations, a numerical method RK4 is now used, and for the conformation and validation Homotopy Asymptotic Method (HAM) is also applied.

Furthermore, the current work is also matched with published literature and outstanding agreement is found.

\section{Result and discussion}

The heat and mass transfer across an unsteady rotational inclined plane using 3D thinfilm nanomaterial flow has been investigated. The findings were acquired using the numerical approach Runge-Kutta fourth order method (RK4), while the analytical solution for the validation purposes is obtained using Homotopy Asymptotic Method (HAM). Figure 1 depicts the current problem physical configuration. Figures 2.,3.,4.,5 display the impact of $S$ on axial as well as radial velocities, drainage moment, and induced moment, respectively. The variation in fluid moment is depicted by increased quantities of said unsteadiness factor $S$.

For greater values of unsteadiness factor $S$, the momentum thickness grows, and as a consequence, most of the said kinds of fluid flow fall, as seen in the depicted graphs. Figure 6 illustrates that the temperature distribution becomes substantially decreases with the increasing values of parameter $S$. In reality, as the values of $S$ increases, heat transmission rate from the disc to the flowing fluid reduces. Internal collisions of liquid particles are physically hampered at a low rate.

Because as unsteadiness factor $S$ is increased, the boundary layer momentum increases, as a consequence, the concentration field also enhances, as seen in figure 7. 
Figure 8 depicts the variation of Nusselt number effects by unsteadiness factor $S$. It is rich from Fig. 8 that the momentum boundary layer is cooled when the parameter $S$ is increased, as a consequence local Nusselt number rises. As illustrated from figure 9, that Sherwood number decreases as the parameter $S$ increases because of inter collision of the microscopic fluid particles.

As depicted in figure 10, large amount of $N t$ and $N b$, enhances heat transfer rate. Indeed, higher Brownian motion factor $\mathrm{Nb}$ upsurges the thickness of thermal boundary layer.

Figure 11 demonstrates that how concentration rate decreases with changing of Schmidt number Sc. In fact, increasing the $S c$ parameter enhances kinematic viscosity and increases chemical species concentration, lowering the Sherwood number.

Figure 12 depicts the effect of $\operatorname{Pr}$ (Prandtl number), on the heat flux. Thermal boundary layer thickness reduces with the enhances of $\mathrm{Pr}$, and so as a consequence, the cooling rate is decreased.

The graphical comparison for the RK4 and HAM methods are sketched in figures 13-16 for the axial and drainage velocities, temperature and concentration fields, respectively. From both the methods we get outstanding agreement. Furthermore, the numerical results of the RK4 and HAM methods for the Nusselt number and Sherwood number are given in table 1. Furthermore, we verified the acquired results by establishing a comparing with previous literatures, and we discovered an outstanding match, confirming the accuracy of the current communication as shown in table 2.

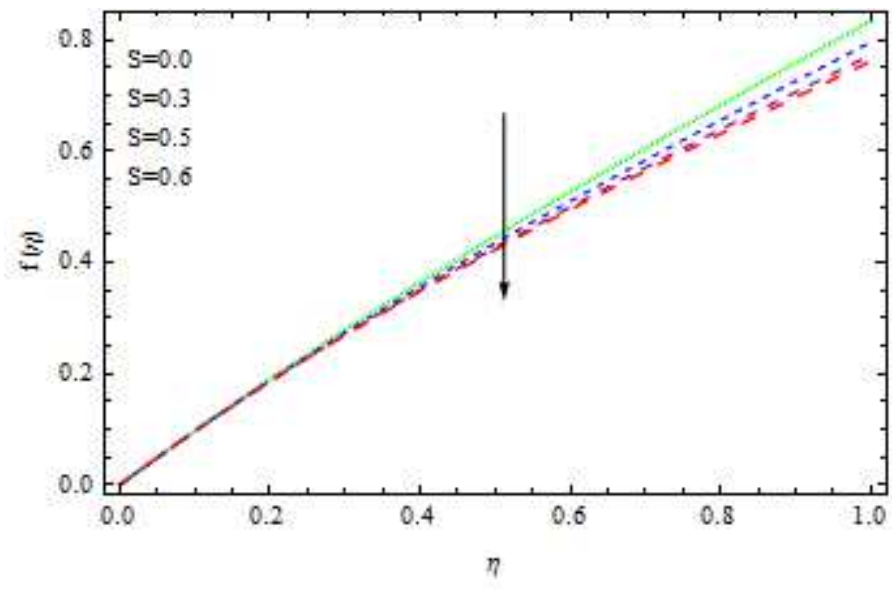

Figure. 2. The axial velocity affected by $\mathrm{S}$. 


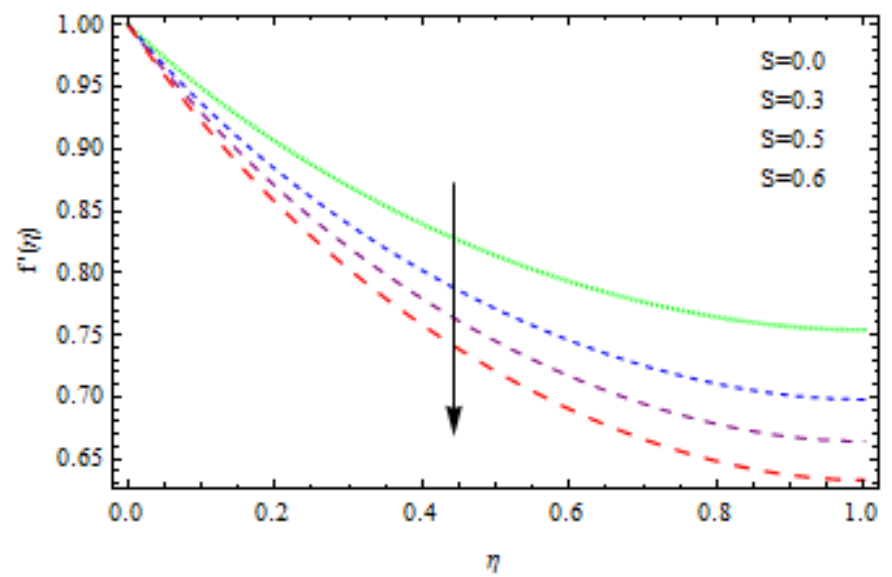

Figure. 3. The radial velocity affected by S.

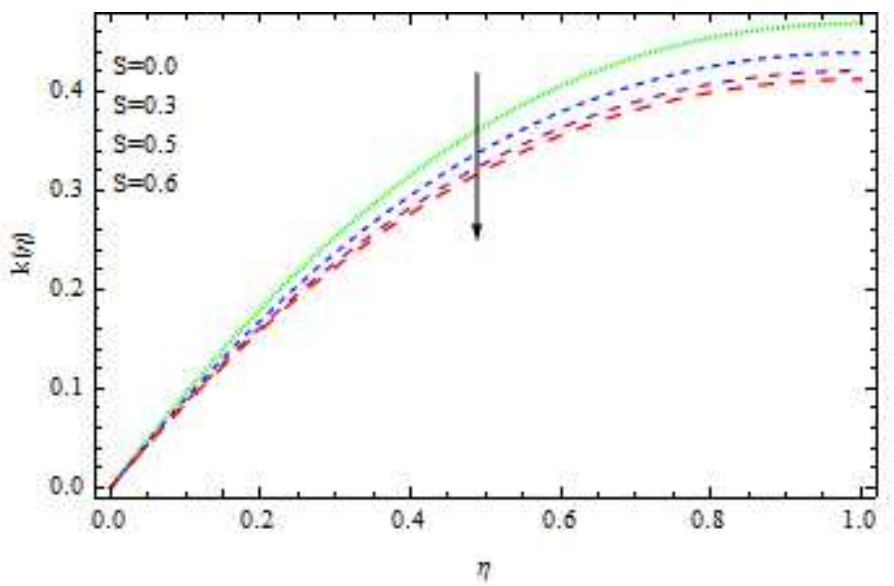

Figure. 4. The $\mathrm{x}$-direction draining flow affected by $\mathrm{S}$.

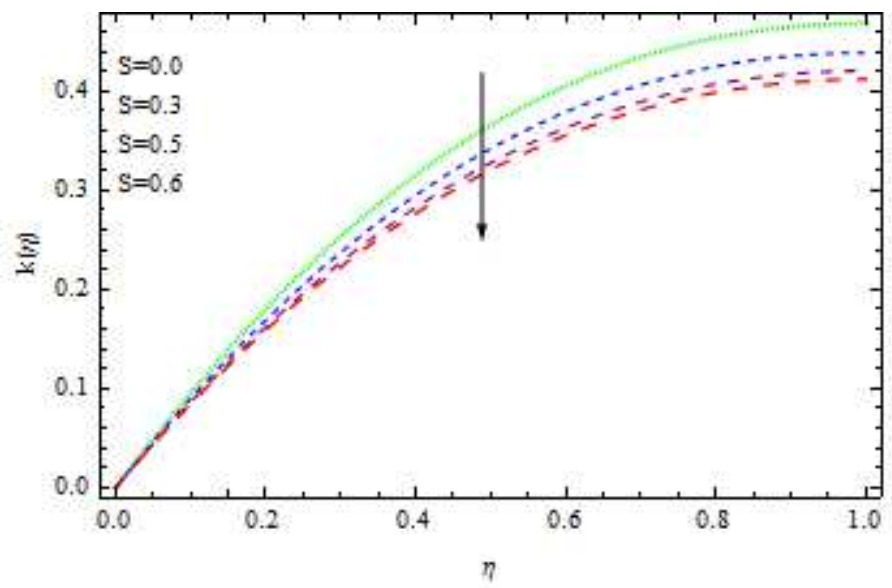

Figure. 5. The y-direction induced flow affected by S. 


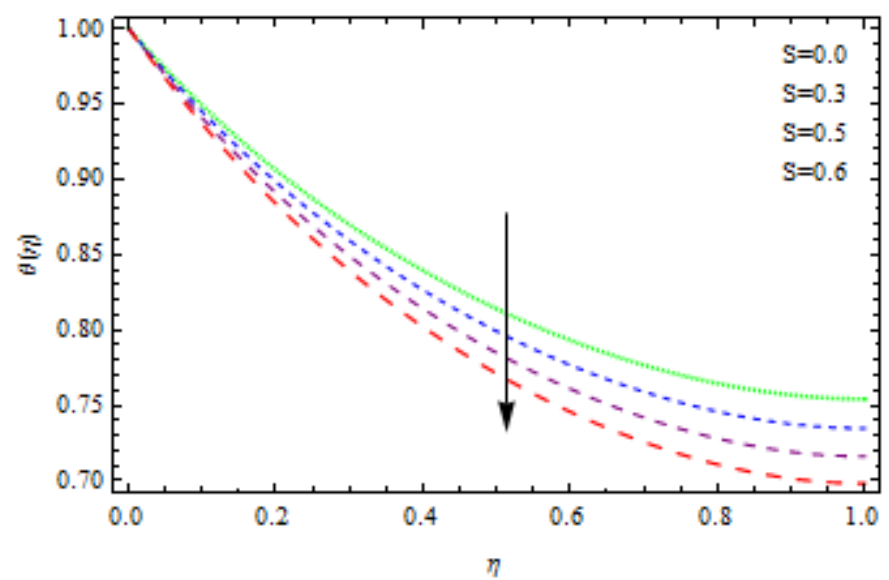

Figure. 6. Temperature field affected by S.

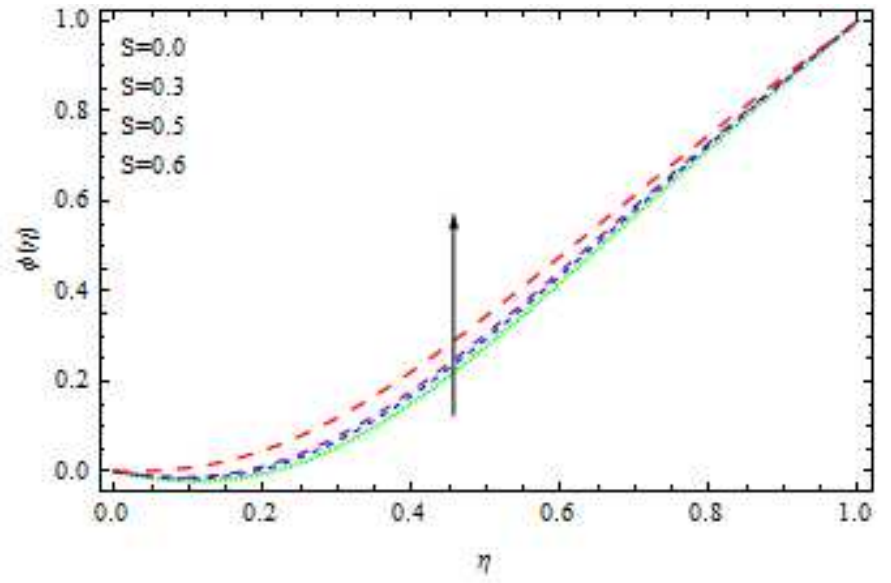

Figure. 7. Concentration field affected by S.

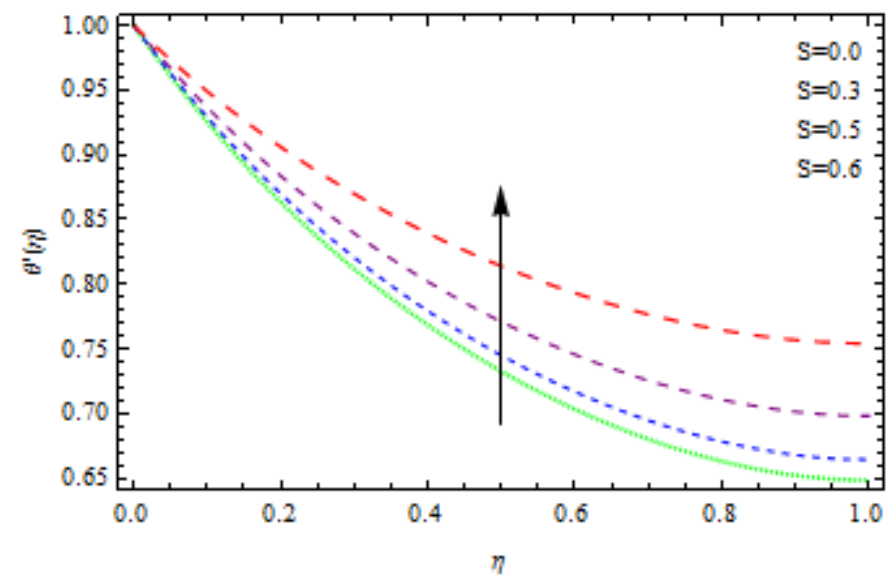

Figure. 8. Heat transmission affected by S. 


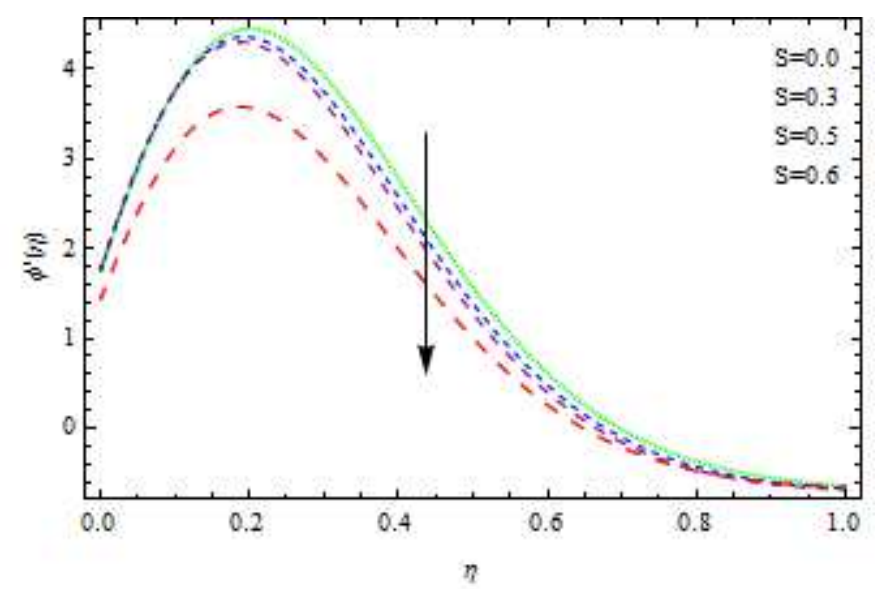

Figure. 9. Sherwood number affected by S.

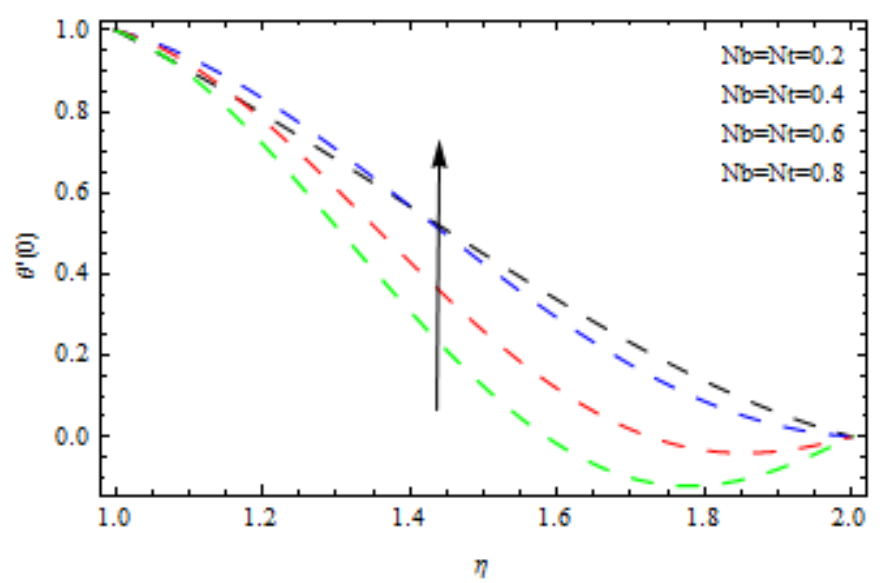

Figure. 10. Heat transmission affected by $\mathrm{Nt}$ and $\mathrm{Nb}$.

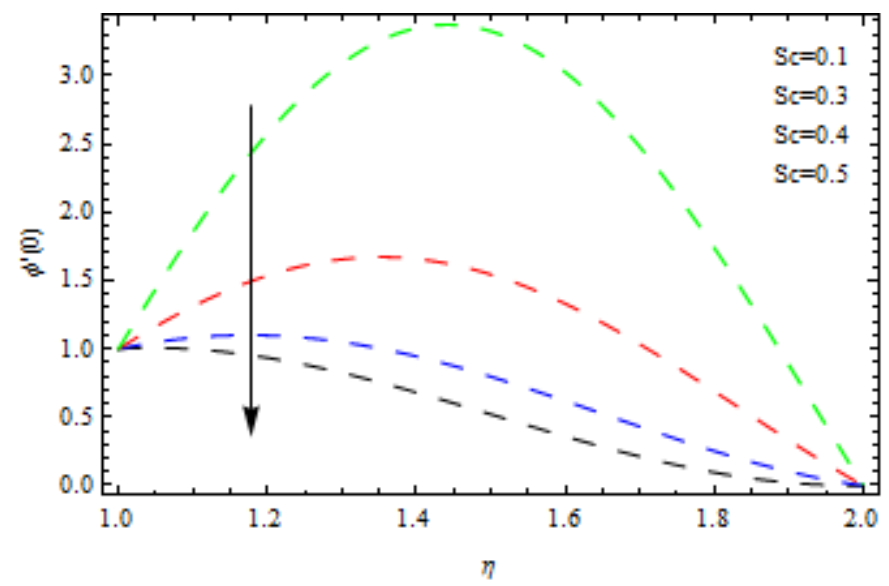

Figure. 11. The Sherwood number affected by Sc. 


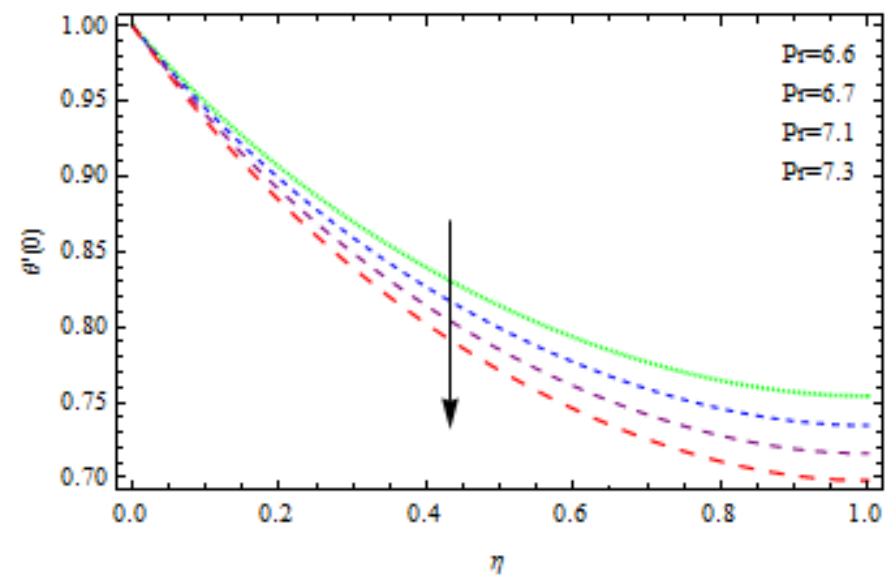

Figure. 12. Heat transmission affected by Pr.

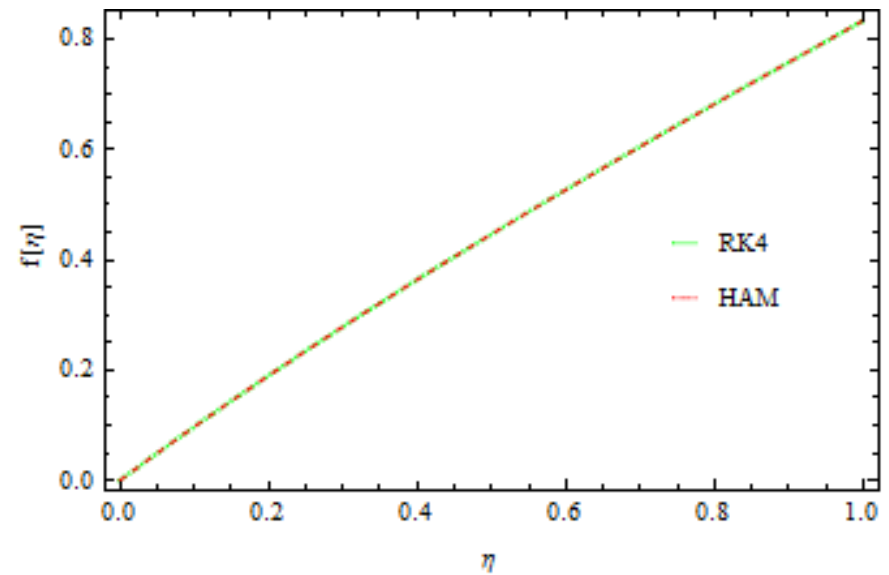

Figure. 13. Comparison of RK4 and HAM on the axial velocity.

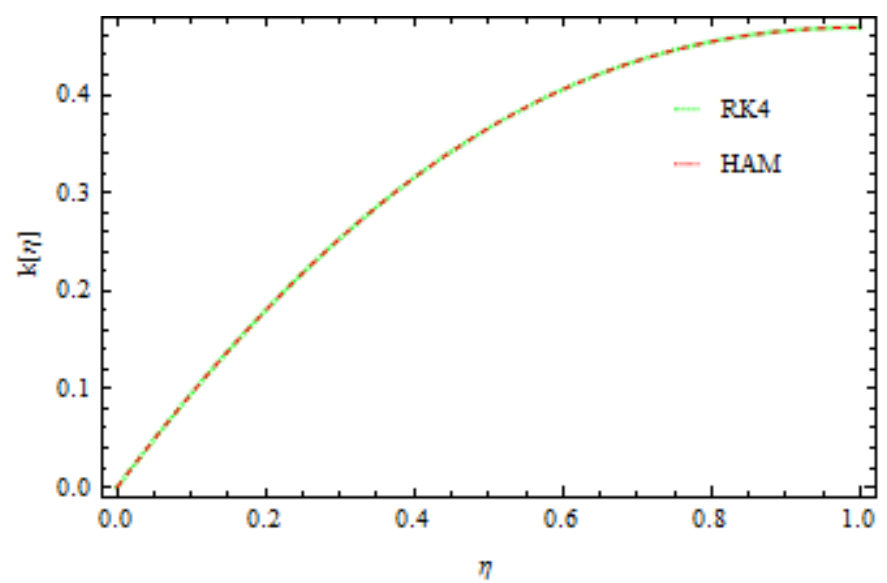


Figure. 14. Comparison of RK4 and HAM on the drainage flow.

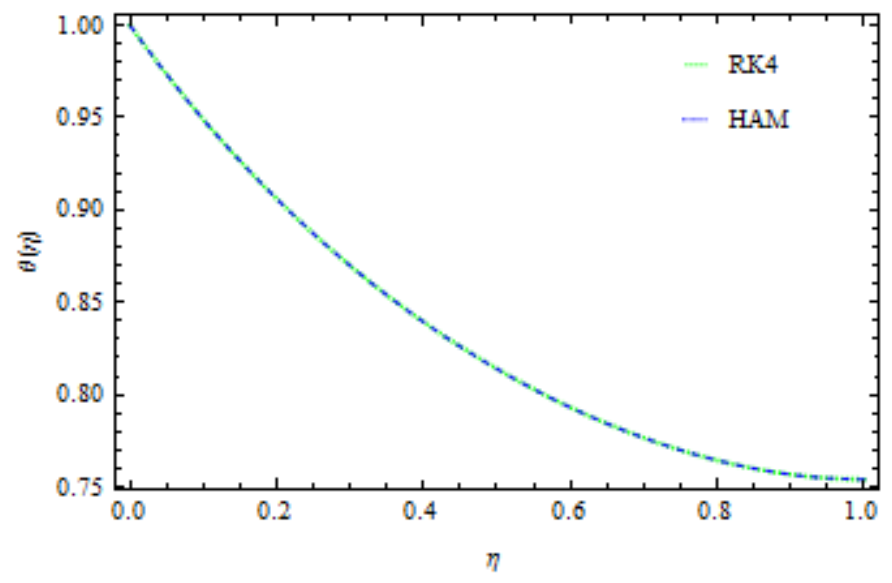

Figure. 15. Temperature comparison of RK4 and HAM.

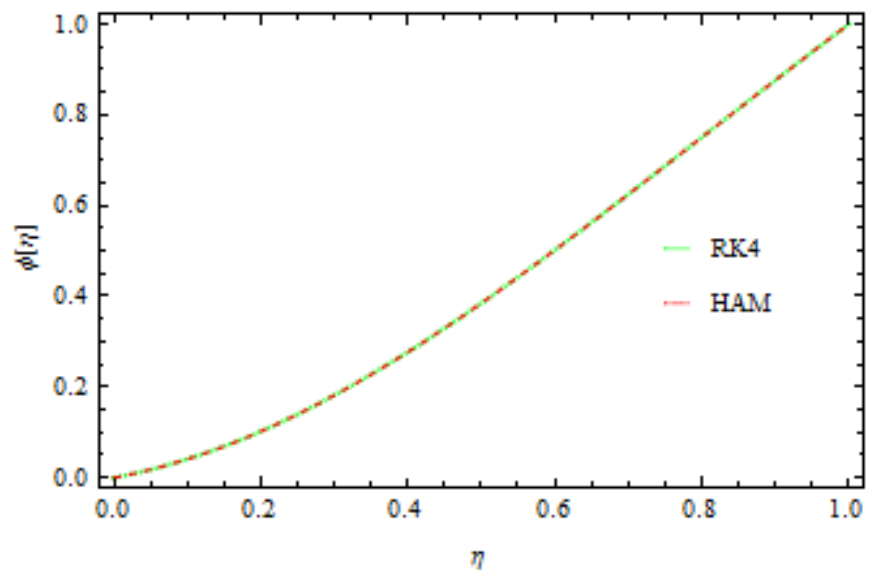

Figure. 16. Concentration flow comparison of RK4 and HAM.

Table 1. The comparison of the present work with published work reported by Gul. Et al. [13] with respect to the heat transfer rate and concentration rate, when $\operatorname{Pr}=6, N t=$ $N b=S c=S=0.7 . S=0.4$ 


\begin{tabular}{|c|c|c|c|c|}
\hline$\eta$ & $\begin{array}{c}\text { RK4 } \\
-\theta^{\prime}(0)\end{array}$ & $\begin{array}{c}\text { HAM } \\
-\theta^{\prime}(0)\end{array}$ & $\begin{array}{c}\text { RK4 } \\
-\phi^{\prime}(0)\end{array}$ & $\begin{array}{c}\text { HAM } \\
-\phi^{\prime}(0)\end{array}$ \\
\hline 0.1 & 0.9048 & 0.9046 & 1.0947 & 1.0921 \\
\hline 0.2 & 0.8180 & 0.8180 & 1.1790 & 1.1790 \\
\hline 0.3 & 0.7399 & 0.7398 & 1.2509 & 1.2502 \\
\hline 0.4 & 0.6711 & 0.6713 & 1.3081 & 1.3082 \\
\hline 0.5 & 0.6127 & 0.6127 & 1.3480 & 1.3480 \\
\hline 0.6 & 0.5661 & 0.5661 & 1.3683 & 1.3682 \\
\hline 0.7 & 0.5330 & 0.5330 & 1.3662 & 1.3661 \\
\hline 0.8 & 0.5161 & 0.5161 & 1.3386 & 1.3385 \\
\hline 0.9 & 0.5198 & 0.5197 & 1.2805 & 1.2812 \\
\hline 1.0 & 0.5522 & 0.5501 & 1.1831 & 1.1825 \\
\hline
\end{tabular}

Table 2. The comparison of current work with available work reported by Sheikholeslami al. [4] for $-\theta^{\prime}(0)$ and $-\phi^{\prime}(0)$ fixing $P r=6.5, N t=N b=S c=S=0.9 . S=0.6$

\begin{tabular}{|c|c|c|c|c|}
\hline$\eta$ & $\begin{array}{c}\text { RK4 } \\
-\theta^{\prime}(0)\end{array}$ & $\begin{array}{c}\text { Ref. [4] } \\
-\theta^{\prime}(0)\end{array}$ & $\begin{array}{c}\text { RK4 } \\
-\phi^{\prime}(0)\end{array}$ & $\begin{array}{c}\text { Ref. [4] } \\
-\phi^{\prime}(0)\end{array}$ \\
\hline 0.1 & 0.9137 & 0.9046 & 1.0936 & 1.0921 \\
\hline 0.2 & 0.8271 & 0.8180 & 1.1791 & 1.1790 \\
\hline 0.3 & 0.7261 & 0.7398 & 1.2510 & 1.2502 \\
\hline 0.4 & 0.6620 & 0.6713 & 1.3084 & 1.3082 \\
\hline 0.5 & 0.6115 & 0.6127 & 1.3471 & 1.3480 \\
\hline 0.6 & 0.5741 & 0.5661 & 1.3678 & 1.3682 \\
\hline
\end{tabular}




\begin{tabular}{|c|c|c|c|c|}
0.7 & 0.5229 & 0.5330 & 1.3660 & 1.3661 \\
\hline 0.8 & 0.5006 & 0.5161 & 1.3375 & 1.3384 \\
\hline 0.9 & 0.5011 & 0.5197 & 1.2806 & 1.2812 \\
\hline 1.0 & 0.5503 & 0.5501 & 1.1827 & 1.1825 \\
\hline
\end{tabular}

\section{Conclusion}

The existing literature focuses primarily on two-dimensional flow problems. The pouring of 3D nanomaterial's across a stretchable inclined rotatable frame is investigated in this paper. The following is a summary of the new findings in the Numerical and analytical solutions:

1. As the dimensionless parameter $\mathrm{S}$ raises, the temperature field decreases. In reality, as the values of $S$ increases, heat transmission rate from the disc to the flowing fluid reduces. Internal collisions of liquid particles are physically hampered at a low rate.

2. The momentum boundary layer is cooled when the parameter $S$ is increased, as a consequence local Nusselt number rises.

3. Sherwood number decreases as the parameter $S$ increases because of inter collision of the microscopic fluid particles.

4. Enhancing in the apparent viscosity and concentrations of the chemical reactions, a higher Schmidt number, Sc, lowers the Sherwood number.

5. With increasing values of Prandtl number the Nusselt number decreases. 


\section{References}

1. Sparrow, E.M., Gregg, J.L. Discussion: "A Theory of Rotating Condensation. Heat Transf. 1959, 81, 113-119.

2. Beckett, P.M., Hudson, P.C., Poots, G. Laminar film condensation due to a rotating disk J. Eng. Math. 1973, 7, 63-73.

3. Chary, S.P., Sarma, P.K. Condensation on a Rotating Disk With Constant Axial Suction J. Heat Transf. 1976, 98, 682-684.

4. Sheikholeslami, M., Hatami, Ganji, D.D. Numerical investigation of nanofluid spraying on an inclined rotating disk for cooling process J. Mol. Liq. 2015, 211, 577-583.

5. Attia, H.A., Fluid Dyn. Effect of Hall current on the unsteady MHD flow due to a rotating disk with uniform suction or injection, Res. 1998, 23, 1089-1098.

6. Bachok, N., Ishak, A., Pop, I. Unsteady boundary layer flow of a nanofluid over a permeable stretching/shrinking sheet, Int. J. Heat Mass Transfer, 2012, 55, 2102-2109.

7. Freidoonimehr, N., Rashidi, M.M., Mahmud, S. Numerical investigation of velocity slip and temperature jump effects on unsteady flow over a stretching permeable surface Int. J. Therm. Sci. 2015, 87, 136.

8. Makinde, O.D., Mabood, F., Khan, W.A., Tshehla, M.S. MHD flow of a variable viscosity nanofluid over a radially stretching convective surface with radiative heat $\mathrm{J}$. Mol. Liq. 2016, 219, 624-630.

9. Akbar, T., Batool, S., Nawaz, R., Zia, Q.M.Z. Magnetohydrodynamics flow of nanofluid due to stretching/shrinking surface with slip effect, Adv. Mech. Eng. 2017, 9, 1.

10. Ramzan, M., Chung, J.D., Ullah, N. Partial slip effect in the flow of MHD micropolar nanofluid flow due to a rotating disk - A numerical approach Results Phys. 2017, 7, 3557-3566.

11. Abdullah, A.A.A., Kalidasan, K., Kolsi, L., Aydi, Malekshah, A.K., Hussein, Kanna, P.R. Three-dimensional investigation of the effects of external magnetic field inclination on laminar natural convection heat transfer in CNT-water nanofluid filled cavityJ. Mol. Liq. 2018, 252, 454-468.

12. Ali, S.A., Gul, T., A convective study of $\mathrm{Al} 2 \mathrm{O} 3-\mathrm{H} 2 \mathrm{O}$ and $\mathrm{Cu}-\mathrm{H} 2 \mathrm{O}$ nano-liquid films sprayed over a stretching cylinder with viscous dissipation, Eur. Phys. J. Plus , 2017, 132, 495.

13. T. Gul, M.Sohil, Theoritical and Applied mehanics letter. Marangoni liquid film scattering over an extending cylinder. 2019, 9, 106-112.

14. Choi, S.U.S. Enhancing thermal conductivity of fluids with nanoparticles. In Developments and Applications

of Non-Newtonian Ows; Siginer, D.A., Wang, H.P., Eds.; ASME: New York, NY, USA, 1995; Volume 66,

pp. 99-105.

15. Yu, W.; Xie, H.Q.; Chen, L.F.; Li, Y. Investigation on the thermal transport properties of ethylene glycol-based

nanofluids containing copper nanoparticles. Powder Technol. 2010, 197, 218-221.

16. Yu, W.; Xie, H.Q.; Li, Y.; Chen, L.F. Experimental investigation om the heat transfer properties of $\mathrm{Al}$

$2 \mathrm{O} 3$

nanofluids using the mixture of ethylene. Powder Technol. 2012, 230, 14-19. 
17. Chen, L.F.; Yu, W.; Xie, H.Q.; Li, Y. Enhanced thermal conductivity of nanofluids containing Ag/MWNT

composites. Powder Technol. 2012, 231, 18-20.

18. Xie, H.Q.; Chen, L.F.J. Review on the preparation and thermal performances of carbon nanotube contained

nanofluids. Chem. Eng. Data 2011, 56, 1030-1041.

19. Yu, W.; Xie, H.Q.; Chen, W. Experimental investigation on thermal conductivity of nanofluids containing

graphene oxide nanosheets. J. Appl. Phys. 2010, 107, 094317.

20. Xiao, B.; Yang, Y.; Chen, L. Developing a novel form of thermal conductivity of nanofluids with Brownian

motion effect by means of fractal geometry. Powder Technol. 2013, doi:10.1016/j.powtec.2013.02.029

21. Cai, J.; Hu, X.; Xiao, B.; Zhou, Y.; Wei, W. Recent developments on fractal-based approaches to nanofluids

and nanoparticles aggregation. Int. J. Heat Mass Transf. 2017, 105, 623-637.

22. Buongiorno, J. Convective transport in nanofluids. ASME J. Heat Transf. 2006, 128, 240-250.

23. Ellahi, R. The effects of MHD and temperature dependent viscosity on the flow of a non-Newtonian nanofluid

in a pipe: Analytical solution. Appl. Math. Model. 2013, 37, 1451-1457.

24. Khan, W.A.; Pop, I. Boundary layer flow of a nanofluid past a stretching sheet. Int. J. Heat Mass Transf. 2010,

$53,2477-2483$.

25. Mustafa, M.; Hina, S.; Hayat, T.; Alsaedi, A. Influence of wall properties on the peristaltic flow of a nanofluid:

Analytic and numerical solutions. Int. J. Heat Mass Transf. 2012, 55, 4871-4877.

26. Akbar, N.S.; Nadeem, S. Endoscopic effects on peristaltic flow of a nanofluid. Commun. Theor. Phys. 2011, 56,

761-768.

27. Nowar, K. Peristaltic flow of a nanofluid under the effect of Hall current and porous medium. Hindawi Publ.

Corp. Math. Probl. Eng. 2014, 2014, doi:10.1155/2014/389581.

28. Choi, S.U.S.; Zhang, Z.G.; Yu, W.; Lockwood, F.E.; Grulke, W.A. Anomalously thermal conductivity

enhancement in nanotube suspensions. Appl. Phys. Lett. 2001, 79, 2252-2254.

29. Terekhov, V.I.; Kalinina, S.V.; Lemanov, V.V. The mechanism of heat transfer in nanofluids, State of the Art

(Review): Part 1. Synthesis and properties of Nanofluids. Thermophys. Aeromech. 2010, $17,1-4$.

30. Yu, W.; France, D.M.; Routbort, J.L.; Choi, S.U.S. Review and comparison of nanofluids thermal conductivity

and heat transfer enhancements. Heat Transf. Eng. 2008, 29, 432-460.

31. Hojjat, M.; Etmat, S.G.; Bagheri, R.; Thibault, J. Laminar convective heat transfer of Non-Newtonian

nanofluids with constant wall temperature. Heat Mass Transf. 2011, 47, 203-209 
32. He, Y.; Men, Y.; Liu, X.; Lu, H.; Chen, H.; Ding, Y. Study on forced convective heat transfer of Non-Newtonian

nanofluids. J. Therm. Sci. 2009, 18, 20-26.

33. Polidoiri, G.; Fohanno, S.; Nguyen, C.T. A note on heat transfer modeling of Newtonian nanofluids in

laminar free convection. Int. J. Therm. Sci. 2007, 46, 739-744.

34. Lakshmisha, K.N.; Venkatswaran, S.; Nath, G. Three-Dimensional unsteady flow with heat and mass transfer

over a continuous stretching surface. J. Heat Transf. 1988, 110, 590-595.

35. Wang, C.Y. The three-dimensional unsteady flow due to a stretching flat surface. Phys. Fluids 1984, 27,

1915-1917.

36. Ahmad, S.; Rohni, A.M.; Pop, I. Blasius and Sakiadis problems in nanofluids. Acta Mech. 2011, 218, 195-204.

37. Chamkha, A.J.; Aly, A.M.; Al Mudhaf, H. Laminar MHD mixed convection flow of a nanofluid along a stretching permeable surface in the presence of heat generation or absorption effects. Int. J. Microscale Nanoscale Therm. Fluid Transp. Phenom. 2011, 2, 51-70.

37. Kandasamy, R.; Loganathan, P.; Puvi Arasu, P. Scaling group transformation for MHD boundary layer flow

of a nanofluid past a vertical stretching surface in the presence of suction and injection. Nuclear Eng. Des. 2011, 241, 2053-2059.

38. Sakiadis, B.C. Boundary layer behavior on continuous solid surface: Boundary layer on a continuous flat

surface. Am. Inst. Chm. Eng. J. 1961, 7, 213-215.

39. Crane, L.J. Flow past a stretching sheet. Zeits für Ange Math. Phys. 1970, 21, 645647.

40. Vajravelu, K.; Rollins, D. Hydromagnetic flow of a second grade fluid over a stretching sheet. Appl. Maths.

Comput. 2004, 148. 783-791.

41. Abu-Nada, E. Effects of variable viscosity and thermal conductivity of Al2O3 water nanofluid on heat transfer enhancement in natural convection. Int. J. Heat Fluid Flow 2009, 30, 679-690.

42. Nasrin, R.; Alim, M.A. Entropy generation by nanofluid with variable thermal conductivity and viscosity in a flat plate solar collector. Int. J. Eng. Sci. Technol. 2015, 7, 80-93.

43. Khan, Y.; Wua, Q.; Faraz, N.; Yildirim, A. The effects of variable viscosity and thermal conductivity on a thin film flow over a shrinking/stretching sheet. Comput. Math. Appl. 2011, 61, 3391-3399.

44. Khan, N.S.; Gul, T.; Islam, S.; Khan, W.; Khan, I.; Ali, L. Thin film flow of a secondgrade fluid in a porous medium past a stretching sheet with heat transfer. Alex. Eng. J. 2017, in press.

45. Khan, N.S.; Gul, T.; Islam, S.; Khan, W. Thermophoresis and thermal radiation with heat and mass transfer in a magnetohydrodynamic thin film second-grade fluid of variable properties past a stretching sheet. Eur. Phys. J. Plus 2017, 132, doi:10.1140/epjp/i2017-11277-3. 
46. Aziz, R.C.; Hashim, I.; Alomari, A.k. Thin film flow and heat transfer on an unsteady stretching sheet with internal heating. Meccanica 2011, 46, 349-357.

47. Khan, W.; Gul, T.; Idrees, M.; Islam, S.; Dennis, L.C.C. Thin film Williamson nanofluid flow with varying viscosity and thermal conductivity on a time-dependent stretching sheet. Appl. Sci. 2016, 6, 334.

48. Qasim, M.; Khan, Z.H.; Lopez, R.J.; Khan, W.A. Heat and mass transfer in nanofluid over an unsteady stretching sheet using Buongiorno's model. Eur. Phys. J. Plus 2016, 131, 1-16.

49. Prashant, G.M.; Jagdish, T.; Abel, M.S. Thin film flow and heat transfer on an unsteady stretching sheet with thermal radiation, internal heating in presence of external magnetic field. arXiv:1603.3664 physics. flu-dyn.

2016, 3, 1-16.

50. Kumari, M.; Gireesha, B.J.; Gorla, R.S.R. Heat and mass transfer in nanofluid over an unsteady stretching surface. J. Nanofluids 2015, 4, 1-8.

51. Wang, C.Y. Fluid flow due to a stretching cylinder. Phys. Fluids 1988, 31, 466-468. 52. Ishak, A.; Nazar, R.; Pop, I. Magnetohydrodynamic (MHD) flow and heat transfer due to a stretching cylinder. Energ. Convers. Manag. 2008, 49, 3265-3269.

53. Wang, C.Y. Natural convection on a cylinder. Commun. Nonlinear Sci. Numer. Simul. 2012, 17, 1098-1103.

54. Elbashbeshy, E.M.A.; Emam, T.G.; El-Azab, M.S.; Abdelgaber, K.M. Effect of magnetic field on flow and heat transfer over a stretching cylinder in the presence of a heat source/sink with suction/injection. J. App. Mech. Eng. I 2012, 106, doi:10.4172/2168-9873.1000106.

55. Ashorynejad, H.R.; Sheikoleslami, M.; Pop, I.; Ganji, D.D. Nanofluid flow and heat transfer due to a stretching cylinder in the presence of magnetic field. Heat Mass Transf. 2012, 49, 427-436.

56. Rangi, R.R.; Ahmad, N. Boundary layer flow past a stretching cylinder and heat transfer with variable thermal conductivity. Appl. Math. 2012, 3, 205-209.

57. Sheikholeslami, M. Effect of uniform suction of nanofluid flow and heat transfer over a cylinder. Braz. Soc. Mech. Sci. Eng. 2014, doi:10.1007/40430-014-0242-z.

58. Wang, C.Y. Liquid film sprayed on on a stretching cylinder. Chem. Eng. Commun. 2006, 193, 869-878.

59. Koo, J.; Kleinstreuer, C. Viscous dissipation effects in micro tubes and micro channels. Int. J. Heat Mass Transf. 2004, 47, 3159-3169.

60. Koo, J. Computational Nanofluid Flow and Heat Transfer Analysis Applied to Microsystems. Ph.D. Thesis, NC State University, Raleigh, NC, USA, 2004.

61. Prasher, R.S.; Bhattacharya, P.; Phelan, P.E. Thermal conductivity of nano scale colloidal solution. Phys. Rev. Lett. 2005, 94, 025901.

62. Jang, S.P.; Choi, S.U.S. The role of Brownian motion in the enhanced thermal conductivity of nanofluids. Appl. Phys. Lett. 2004, 84, 4316-4318.

63. Li, J. Computational Analysis of Nanofluid Flow in Micro Channels with Applications to Micro-Heat Sinks and Bio-MEMS. Ph.D. Thesis, NC State University, Raleigh, NC, USA, 2008.

64. Koo, J.; Kleinstreuer, C. Laminar nanofluid flow in micro-heat-sinks. Int. J. Heat Mass Transf. 2005, 48, 2652-2661. 
65. Brinkman, H.C. The viscosity of concentrated suspensions and solutions. J. Chem. Phys. 1952, 20, 571.

66. Einstein, A. Investigation on the Theory of Brownian Motion; Dover Publications, Inc.: New York, NY, USA, 1956. 